Journal of Applied Analysis

Vol. 12, No. 1 (2006), pp. 127-143

\title{
PRODUCTS OF STRONG ŚWIA̧TKOWSKI FUNCTIONS
}

\author{
P. SZCZUKA \\ Received November 10, 2004 and, in revised form, March 10, 2005
}

\begin{abstract}
In this paper we characterize the products of four or more strong Świạtkowski functions.
\end{abstract}

\section{Preliminaries}

The letters $\mathbb{R}$ and $\mathbb{N}$ denote the real line and the set of positive integers, respectively. For all $a, b \in \mathbb{R}$, we define $\mathrm{I}(a, b] \stackrel{\mathrm{df}}{=}(a, b]$, if $a<b$, and $\mathrm{I}(a, b] \stackrel{\mathrm{df}}{=}[b, a)$ otherwise. The symbol $\mathrm{I}(a, b)$ is defined analogously.

For each $A \subset \mathbb{R}$ we use the symbols int $A, \operatorname{cl} A, \operatorname{bd} A$ and card $A$ to denote the interior, the closure, the boundary, and the cardinality of $A$, respectively. We say that a set $A \subset \mathbb{R}$ is simply open [1], if it can be written as the union of an open set and a nowhere dense set.

Let $f: I \rightarrow \mathbb{R}$, where $I$ is a nondegenerate interval. The symbol $\mathcal{C}(f)$ stands for the set of all points of continuity of $f$. We say that $f$ is a Darboux function, if it maps connected sets onto connected sets. We say that $f$ is quasi-continuous in the sense of Kempisty [4], if for all $x \in I$ and open sets $U \ni x$ and $V \ni f(x)$, the set $\operatorname{int}\left(U \cap f^{-1}(V)\right)$ is nonempty. We say that

2000 Mathematics Subject Classification. 26A15, 54C30.

Key words and phrases. Strong Świątkowski function, product of functions.

Supported by Kazimierz Wielki University.

ISSN 1425-6908（C) Heldermann Verlag. 
$f$ is cliquish [10], if the set of points of continuity of $f$ is dense in $I$. We say that $f$ is a strong Światkowski function $[6]\left(f \in \mathcal{S}_{s}\right)$, if whenever $\alpha, \beta \in I$ and $y \in \mathrm{I}(f(\alpha), f(\beta))$, there is an $x_{0} \in \mathrm{I}(\alpha, \beta) \cap \mathcal{C}(f)$ such that $f\left(x_{0}\right)=y$.

One can easily see that strong Świątkowski functions are both Darboux and quasi-continuous. The symbol $[f=a]$ stands for the set $\{x \in I: f(x)=a\}$. Similarly we define the symbols $[f<a],[f>a]$, etc. The symbol $\mathcal{M}$ denotes the class of all real functions $f$ defined on a nondegenerate interval such that $f$ has a zero in each subinterval in which it changes sign.

\section{Introduction}

In 1996 Maliszewski proved the following theorem [7].

Theorem 2.1. For each function $f$ the following conditions are equivalent:

i) $f$ is a finite product of Darboux quasi-continuous functions,

ii) $f \in \mathcal{M}, f$ is cliquish, and the set $[f=0]$ is simply open,

iii) there are Darboux quasi-continuous functions $g$ and $h$ such that $f=g h$.

He showed also that there is a bounded Darboux quasi-continuous function which cannot be written as the finite product of strong Świątkowski functions [7, Proposition III.4.1]. Moreover he remarked that the sign function can be written as the product of three strong Świątkowski functions but it cannot be written as the product of two strong Świątkowski functions [7, Propositions III.4.2 and III.4.3].

In 2003 I showed that there is a function which can be written as the product of four strong Świątkowski functions, and which cannot be written as the product of three strong Świątkowski functions [9]. In this paper I characterize products of four or more strong Świątkowski functions. However, the following problem is still open.

Problem 1. Characterize the products of two strong Swiatkowski functions and the products of three strong Światkowski functions.

\section{Auxiliary lemmas}

Lemmas 3.1 and 3.2 can be easily proved using [5, Theorem 12].

Lemma 3.1. Let $I \subset \mathbb{R}$ be an interval, $g: I \rightarrow \mathbb{R}$ and $x \in I$. If $g\lceil I \cap$ $(-\infty, x) \in \dot{\mathcal{S}}_{s}, g\left\lceil I \cap(x, \infty) \in \dot{\mathcal{S}}_{s}\right.$ and $x \in \mathcal{C}(g)$, then $g \in \dot{\mathcal{S}}_{s}$. 
Lemma 3.2. Let $I \subset \mathbb{R}$ be an interval, $g: I \rightarrow \mathbb{R}$ and $x \in I$. If $g\lceil I \cap$ $(-\infty, x] \in \mathcal{S}_{s}, g\left\lceil I \cap(x, \infty) \in \mathcal{S}_{s}\right.$ and $g(x) \in g[[x, t] \cap \mathcal{C}(g)]$ for each $t \in$ $(x, \sup I)$, then $g \in \mathcal{S}_{s}$.

The next lemma is interesting in itself.

Lemma 3.3. Let $I \subset \mathbb{R}$ be an interval, $g: I \rightarrow \mathbb{R}$ and $h: \mathbb{R} \rightarrow \mathbb{R}$. If $g, h \in \dot{\mathcal{S}}_{s}$, then $h \circ g \in \dot{\mathcal{S}}_{s}$.

Proof. Let $\alpha, \beta \in I, \alpha<\beta$ and $z \in \mathrm{I}((h \circ g)(\alpha),(h \circ g)(\beta))$. Since $h \in \mathcal{S}_{s}$, there is a $y \in \mathrm{I}(g(\alpha), g(\beta)) \cap \mathcal{C}(h)$ such that $h(y)=z$. Since $y \in \mathrm{I}(g(\alpha), g(\beta))$ and $g \in \dot{\mathcal{S}}_{s}$, there is an $x \in(\alpha, \beta) \cap \mathcal{C}(g)$ with $g(x)=y$. Clearly $x \in \mathcal{C}(h \circ g)$.

Lemma 3.4. Assume $F \subset C$ are closed and $\mathcal{J}$ is a family of components of $\mathbb{R} \backslash C$ such that $C \subset \mathrm{cl} \bigcup \mathcal{J}$. There is a family $\mathcal{J}^{\prime} \subset \mathcal{J}$ such that

i) for each $J \in \mathcal{J}$, if $F \cap$ bd $J \neq \emptyset$, then $J \in \mathcal{J}^{\prime}$,

ii) for each $c \in F$, if $c$ is a right-hand (left-hand) limit point of $C$, then $c$ is a right-hand (respectively left-hand) limit point of the union $\bigcup \mathcal{J}^{\prime}$, iii) $\operatorname{cl} \bigcup \mathcal{J}^{\prime} \subset F \cup \bigcup_{J \in \mathcal{J}^{\prime}} \operatorname{cl} J$.

Proof. Let $\mathcal{P}$ be the family of all components of $\mathbb{R} \backslash F$ and $P \in \mathcal{P}$. One can easily see that there is a family $\mathcal{J}_{P} \subset \mathcal{J}$ such that $\bigcup \mathcal{J}_{P} \subset P$ and the following conditions hold:

$$
\begin{aligned}
& \text { if } \cap C \neq \emptyset \text {, then } \mathcal{J}_{P} \neq \emptyset \text {, } \\
& \text { for each } J \in \mathcal{J} \text {, if } J \subset P \text { and bd } P \cap \text { bd } J \neq \emptyset \text {, } \\
& \text { then } J \in \mathcal{J}_{P} \text {, } \\
& \text { if } \inf P \in \operatorname{cl}(P \cap C) \text {, then } \inf P \in \operatorname{cl} \bigcup \mathcal{J}_{P}, \\
& \text { if } \sup P \in \operatorname{cl}(P \cap C) \text {, then } \sup P \in \operatorname{cl} \bigcup \mathcal{J}_{P} \text {, } \\
& \operatorname{cl} \bigcup \mathcal{J}_{P} \subset \operatorname{bd} P \cup \bigcup_{J \in \mathcal{J}_{P}} \operatorname{cl} J .
\end{aligned}
$$

Define $\mathcal{J}^{\prime}=\bigcup_{P \in \mathcal{P}} \mathcal{J}_{P}$. Clearly $\mathcal{J}^{\prime} \subset \mathcal{J}$. We will show that $\mathcal{J}^{\prime}$ satisfies the conditions i)-iii) of the lemma.

Assume that $F \cap$ bd $J \neq \emptyset$ for some $J \in \mathcal{J}$. Since $F \subset C$, there is a $P \in \mathcal{P}$ with $J \subset P$. Then by (2), $J \in \mathcal{J}_{P} \subset \mathcal{J}^{\prime}$. This proves condition i).

To prove condition ii) assume that $c \in F$ is a right-hand limit point of $C$. We consider two cases. 
If there is a $P \in \mathcal{P}$ with $c=\inf P$, then by (3),

$$
c \in \operatorname{cl} \bigcup \mathcal{J}_{P} \subset \operatorname{cl}\left((c, \infty) \cap \bigcup \mathcal{J}^{\prime}\right) \text {. }
$$

In the opposite case fix a $d>c$. Since $C \subset \operatorname{cl} \cup \mathcal{J}$, we obtain $(c, d) \cap$ $\bigcup \mathcal{J} \neq \emptyset$. By our assumption, there is a $J \in \mathcal{J}$ such that $J \subset(c, d)$ and $(\sup J, d) \cap F \neq \emptyset$. Choose $P \in \mathcal{P}$ with $J \subset P$. Clearly $P \subset(c, d)$.

If $P \cap C=\emptyset$, then $P=J \in \mathcal{J}$, and by (2), $P \in \mathcal{J}_{P} \subset \mathcal{J}^{\prime}$. So, $(c, d) \cap \bigcup \mathcal{J}^{\prime} \neq \emptyset$.

If $P \cap C \neq \emptyset$, then by (1), $\mathcal{J}_{P} \neq \emptyset$. Since $\bigcup \mathcal{J}_{P} \subset P$, we have $(c, d) \cap \bigcup \mathcal{J}^{\prime} \neq$ $\emptyset$. This completes the proof of ii).

Finally we will show iii). Note that by (5),

$$
\begin{aligned}
\operatorname{cl} \bigcup \mathcal{J}^{\prime} & =\operatorname{cl} \bigcup_{P \in \mathcal{P}} \bigcup \mathcal{J}_{P} \subset \mathrm{cl} \bigcup_{P \in \mathcal{P}} \operatorname{bd} P \cup \bigcup_{P \in \mathcal{P}} \operatorname{cl} \bigcup \mathcal{J}_{P} \\
& \subset \operatorname{cl} F \cup \bigcup_{P \in \mathcal{P}}\left(\bigcup_{J \in \mathcal{J}_{P}} \operatorname{cl} J \cup \operatorname{bd} P\right)=F \cup \bigcup_{J \in \mathcal{J}^{\prime}} \operatorname{cl} J .
\end{aligned}
$$

This completes the proof of the lemma.

Lemma 3.5. Let $I=(a, b)$ and $y_{1}, y_{2} \in[0,1]$. There is a strong Światkowski function $\psi: \operatorname{cl} I \rightarrow[0,1]$ such that $\psi[I]=\psi[I \cap \mathcal{C}(\psi)]=(0,1]$, $\psi(a)=y_{1}, \psi(b)=y_{2}$, and $\operatorname{bd} I \subset \mathcal{C}(\psi)$.

Proof. Define the function $\bar{\psi}: \mathbb{R} \rightarrow(0,1]$ by

$$
\bar{\psi}(x)=\left\{\begin{array}{cl}
\min \left\{1, \sin x^{-1}+|x|+1\right\} & \text { if } x \neq 0, \\
2^{-1} & \text { if } x=0 .
\end{array}\right.
$$

Then clearly $\bar{\psi} \in \mathcal{S}_{s}$. Choose elements $a<x_{1}<x_{2}<x_{3}<b$ and define the function $\psi: \operatorname{cl} I \rightarrow[0,1]$ by the formula:

$$
\psi(x)=\left\{\begin{array}{cl}
\bar{\psi}\left(x-x_{2}\right) & \text { if } x \in\left[x_{1}, x_{3}\right] \\
y_{1} & \text { if } x=a \\
y_{2} & \text { if } x=b, \\
\text { linear } & \text { in intervals }\left[a, x_{1}\right] \text { and }\left[x_{3}, b\right] .
\end{array}\right.
$$

One can easily show that the function $\psi$ has all required properties.

Lemmas 3.6-3.11 and Proposition 4.1 will be used in the proof of the main result of this paper. In all of them we assume the following.

$E$ is a compact interval,

$f: E \rightarrow \mathbb{R}$ is cliquish,

$f \in \mathcal{M}$, 
Moreover we define

$$
Y_{f} \stackrel{\mathrm{df}}{=} \operatorname{cl}[f<0] \cap \operatorname{cl}[f>0] .
$$

One can easily see that by (8), $Y_{f} \subset \operatorname{bd}[f=0]$. But $[f=0]$ is simply open, so $Y_{f}$ is nowhere dense.

Lemma 3.6. Let $I \subset E$ be an open interval such that $I \cap \operatorname{int}[f=0]=\emptyset$ and $f=0$ on the set $K \stackrel{\mathrm{df}}{=}\left(Y_{f} \cap I\right) \cup \mathrm{bd} I$. Then there is a strong Swiatkowski function $\varphi: \operatorname{cl} I \rightarrow[-1,1]$ with $\operatorname{sgn} \circ \varphi=\operatorname{sgn} \circ f\lceil\operatorname{cl} I$ such that $\operatorname{bd} I \subset$ $\mathcal{C}(\varphi)$.

Proof. Put $G_{1}=I \cap \operatorname{int}[f<0]$ and $G_{2}=I \cap \operatorname{int}[f>0]$. Let $\mathcal{J}$ be the family of all components of $G_{1} \cup G_{2}$.

For each $J \in \mathcal{J}$, use Lemma 3.5 to construct a strong Świątkowski function $\psi_{J}: \operatorname{cl} J \rightarrow[0,1]$ such that

$$
\begin{aligned}
\psi_{J}[J] & =\psi_{J}\left[J \cap \mathcal{C}\left(\psi_{J}\right)\right]=(0,1], \\
\psi_{J}(x) & =\min \{\operatorname{dist}(K, x) / 2,|\operatorname{sgn} f(x)|\} \quad \text { if } x \in \operatorname{bd} J,
\end{aligned}
$$

and

$$
\text { bd } J \subset \mathcal{C}\left(\psi_{J}\right)
$$

and put

$$
c_{J}=\min \{1, \max \{\operatorname{dist}(K, \inf J), \operatorname{dist}(K, \sup J),|J|\}\},
$$

where $|J|$ denotes the length of the interval $J$.

Define the function $\varphi: \operatorname{cl} I \rightarrow[-1,1]$ by

$$
\varphi(x)= \begin{cases}c_{J} \psi_{J}(x) \operatorname{sgn} f(x) & \text { if } x \in \operatorname{cl} J, J \in \mathcal{J}, \\ \min \{1, \operatorname{dist}(K, x) / 2\} \operatorname{sgn} f(x) & \text { otherwise }\end{cases}
$$

(Notice that if $J_{1}, J_{2} \in \mathcal{J}$ and $\operatorname{cl} J_{1} \cap \operatorname{cl} J_{2}=\{x\}$, then $f(x)=0$. So, $\varphi$ is well defined.)

Clearly $K$ is closed. So, $\operatorname{sgn} \circ \varphi=\operatorname{sgn} \circ f\lceil\operatorname{cl} I$. We will show that $K \subset \mathcal{C}(\varphi)$.

Let $x_{0} \in K \backslash\{\inf I\}$. If there is a $J \in \mathcal{J}$ such that $x_{0}=\sup J$, then by (11), $\varphi$ is continuous from the left at $x_{0}$.

In the opposite case first let $\left(x_{m}\right) \subset\left[\inf I, x_{0}\right) \cap \bigcup \mathcal{J}$ be a sequence convergent to $x_{0}$. For each $m \in \mathbb{N}$ there is a $J_{m}=\left(a_{m}, b_{m}\right) \in \mathcal{J}$ such that $x_{m} \in J_{m}$. Observe that

$$
\lim _{m \rightarrow \infty}\left(a_{m}-b_{m}\right)=0
$$


and $\operatorname{dist}\left(K, b_{m}\right) \leq x_{0}-b_{m}<x_{0}-x_{m}$, whence

$$
\lim _{m \rightarrow \infty} \operatorname{dist}\left(K, b_{m}\right)=0 .
$$

By (12) and (13), we obtain

$$
\lim _{m \rightarrow \infty} \operatorname{dist}\left(K, a_{m}\right)=0
$$

Since

$$
\begin{aligned}
0 & \leq\left|\varphi\left(x_{m}\right)\right|=\left|c_{J_{m}} \psi_{J_{m}}\left(x_{m}\right)\right| \leq c_{J_{m}} \\
& =\min \left\{1, \max \left\{\operatorname{dist}\left(K, a_{m}\right), \operatorname{dist}\left(K, b_{m}\right), b_{m}-a_{m}\right\}\right\},
\end{aligned}
$$

by (12), (13) and (14), we obtain $\lim _{m \rightarrow \infty} \varphi\left(x_{m}\right)=0=\varphi\left(x_{0}\right)$. It proves that $x_{0}$ is a left-hand side point of continuity of the restriction $\varphi \backslash \cup \mathcal{J} \cup\left\{x_{0}\right\}$.

Now put $G_{3}=\operatorname{cl} I \backslash\left(G_{1} \cup G_{2}\right)$. Since $G_{3}=\operatorname{cl}[f=0]=\operatorname{bd}[f=0], G_{3}$ is nowhere dense. Let $\left(x_{m}\right) \subset\left[\inf I, x_{0}\right) \cap G_{3}$ be a sequence convergent to $x_{0}$. Then

$$
\left|\varphi\left(x_{m}\right)\right| \leq \operatorname{dist}\left(K, x_{m}\right) / 2<x_{0}-x_{m} \rightarrow 0=\varphi\left(x_{0}\right) .
$$

So, $x_{0}$ is a left-hand side point of continuity of $\varphi \mid G_{3}$. It follows that $\varphi$ is continuous from the left at $x_{0}$.

Similarly we can prove that the function $\varphi$ is continuous from the right at each point $x_{0} \in K \backslash\{\sup I\}$. So, $K \subset \mathcal{C}(\varphi)$ and in particular bd $I \subset \mathcal{C}(\varphi)$.

Now we will prove that

$$
\forall_{x \in G_{3} \backslash\{\sup J: J \in \mathcal{J}\}} \forall_{\delta>0} \quad \varphi[(x-\delta, x) \cap \mathcal{C}(\varphi)] \supset \mathrm{I}(0, \varphi(x)] .
$$

Indeed, fix an $x \in G_{3} \backslash\{\sup J: J \in \mathcal{J}\}$ and a $\delta>0$. Assume that $\varphi(x)>$ 0 . (The case $\varphi(x)<0$ is analogous.) Then $x \notin K$, so $d \stackrel{\text { df }}{=} \operatorname{dist}(K, x)>0$. Notice that $\varphi(x) \leq \min \{1, d / 2\}$, and define $\delta_{1}=\min \{\varphi(x), \delta\}$. There is a $J \in \mathcal{J}$ such that $\operatorname{cl} J \subset I \cap\left(x-\delta_{1}, x\right)$. For each $z \in I \cap\left(x-\delta_{1}, x\right)$ we have

$$
\operatorname{dist}(K, z) \geq \operatorname{dist}(K, x)-|z-x|>d-\delta_{1} \geq d / 2>0
$$

(in particular, $\operatorname{dist}(K, \inf J)>d / 2$ ) and

$$
\left(x-\delta_{1}, x\right) \cap K=\left(x-\delta_{1}, x\right) \cap Y_{f} \cap I=\emptyset .
$$

Consequently, the function $f$ does not change its sign in the interval $I \cap$ $\left(x-\delta_{1}, x\right)$. But $\operatorname{sgn} \circ \varphi=\operatorname{sgn} \circ f\left\lceil\operatorname{cl} I\right.$, so $\varphi \geq 0$ on $I \cap\left(x-\delta_{1}, x\right)$. Finally since

$$
c_{J} \geq \min \{1, \operatorname{dist}(K, \inf J)\} \geq \min \{1, d / 2\} \geq \varphi(x),
$$

we obtain

$$
\varphi[(x-\delta, x) \cap \mathcal{C}(\varphi)] \supset \varphi[J \cap \mathcal{C}(\varphi)] \supset\left(0, c_{J}\right] \supset(0, \varphi(x)] .
$$

Similarly we can prove that

$$
\forall_{x \in G_{3} \backslash\{\inf J: J \in \mathcal{J}\}} \forall_{\delta>0} \varphi[(x, x+\delta) \cap \mathcal{C}(\varphi)] \supset \mathrm{I}(0, \varphi(x)] .
$$


To complete the proof we will show that $\varphi \in \mathcal{S}_{s}$. Let $c, d \in \operatorname{cl} I, c<d$, and $y \in \mathrm{I}(\varphi(c), \varphi(d))$. Assume that $\varphi(c)<\varphi(d)$. (The case $\varphi(c)>\varphi(d)$ is similar).

If there is a $J \in \mathcal{J}$ such that $c, d \in \operatorname{cl} J$, then since $\psi_{J} \in \mathcal{S}_{s}$, there is an $x_{0} \in(c, d) \cap \mathcal{C}(\varphi)$ such that $\varphi\left(x_{0}\right)=y$. So assume that this case does not hold.

If $y=0$, then put $x_{0}=\sup \{x \in[c, d]: \varphi(x) \leq 0\}$. It suffices to show that $x_{0} \in K \cap(c, d)$.

Indeed, suppose that $x_{0}=d$. Then $d \in \operatorname{cl}[f \leq 0]$. Since $\operatorname{sgn} f(d)=$ $\operatorname{sgn} \varphi(d)=1$ and $I \cap \operatorname{int}[f=0]=\emptyset$, we have $d \in K$ and $f(d)=0$, an impossibility. Similarly we can proceed if $x_{0}=c$. So, $x_{0} \in(c, d)$. Now it is easy to see that $x_{0} \in K$ (recall that $I \cap \operatorname{int}[f=0]=\emptyset$ ).

Finally assume that $y>0$. (The case $y<0$ is analogous.) Then $\varphi(d)>0$. We consider two cases.

Case 1. $d \notin G_{3}$ or $d \in\{\sup J: J \in \mathcal{J}\}$.

Then for some $J \in \mathcal{J}$ we have $d \in \operatorname{cl} J$ and $c \notin \operatorname{cl} J$. If $y \in$ $\mathrm{I}(\varphi(\inf J), \varphi(d))$, then there is an $x_{0} \in(\inf J, d) \cap \mathcal{C}\left(\psi_{J}\right) \subset(c, d) \cap \mathcal{C}(\varphi)$ with $\varphi\left(x_{0}\right)=y$. (Recall that $\left.\psi_{J} \in \dot{\mathcal{S}}_{s}\right)$.

If $y \in(0, \varphi(\inf J)]$, then $\varphi(\inf J)>0$, whence $\inf J \neq \sup J^{\prime}$ for each $J^{\prime} \in \mathcal{J}$. By $(15)$, there is an $x_{0} \in(c, \inf J) \cap \mathcal{C}(\varphi) \subset(c, d) \cap \mathcal{C}(\varphi)$ such that $\varphi\left(x_{0}\right)=y$.

Case 2. $d \in G_{3} \backslash\{\sup J: J \in \mathcal{J}\}$.

Then $y \in(0, \varphi(d))$, so by $(15)$, there is an $x_{0} \in(c, d) \cap \mathcal{C}(\varphi)$ with $\varphi\left(x_{0}\right)=$ $y$.

Remark 3.1. It is quite evident that if $f=0$ on $\operatorname{cl} I$, then there is a function $\varphi: \operatorname{cl} I \rightarrow[-1,1]$, which satisfies all the requirements of Lemma 3.6.

Lemma 3.7. Let $I=(a, b) \subset E$ be an interval such that $f=0$ on $\left(Y_{f} \cap\right.$ $I) \cup\{b\}$. Assume that $I \subset[f=0]$ or $I \cap \operatorname{int}[f=0]=\emptyset$ and $a \in \operatorname{cl}(I \cap$ $[f=0])$. There are strong Światkowski functions $g_{1}, g_{2}: \operatorname{cl} I \rightarrow[-1,1]$ with $\operatorname{sgn} \circ\left(g_{1} g_{2}\right)=\operatorname{sgn} \circ f\left\lceil\operatorname{cl} I\right.$ such that for $i \in\{1,2\}$, we have: $g_{i}(a)=$ $(\operatorname{sgn} f(a))^{i+1} / 2, g_{i}(b)=0, b \in \mathcal{C}\left(g_{i}\right)$, and $g_{i}\left[(a, c) \cap \mathcal{C}\left(g_{i}\right)\right]=[-1,1]$ for each $c \in(a, b)$.

Proof. Choose a sequence $\left(x_{n}\right) \subset I \cap[f=0]$ such that $x_{n} \searrow a$. Put $x_{0}=b$. Define the function $h:(a, b] \rightarrow[-1,1]$ by

$$
h(x)= \begin{cases}0 & \text { if } x=x_{n-1}, n \in \mathbb{N} \\ (-1)^{n-1} & \text { if } x=\left(x_{n-1}+x_{n}\right) / 2, n \in \mathbb{N} \\ \text { linear } & \text { in each interval of the form }\left[x_{n},\left(x_{n-1}+x_{n}\right) / 2\right] \\ & \text { or }\left[\left(x_{n-1}+x_{n}\right) / 2, x_{n-1}\right], n \in \mathbb{N}\end{cases}
$$


Then $h$ is continuous on $(a, b]$. For each $n \in \mathbb{N}$, use Lemma 3.6 to construct a strong Świątkowski function $\varphi_{n}:\left[x_{2 n}, x_{2 n-2}\right] \rightarrow[-1,1]$ with $\operatorname{sgn} \circ \varphi_{n}=$ sgn $\circ f\left\lceil\left[x_{2 n}, x_{2 n-2}\right]\right.$ such that $x_{2 n}, x_{2 n-2} \in \mathcal{C}\left(\varphi_{n}\right)$.

Fix an $i \in\{1,2\}$. Define the function $g_{i}: \operatorname{cl} I \rightarrow[-1,1]$ by

$$
g_{i}(x)= \begin{cases}h(x) & \text { if } x \in \bigcup_{n=1}^{\infty}\left[x_{4 n-2 i+2}, x_{4 n-2 i}\right], \\ \varphi_{2 n+i-2}(x) \operatorname{sgn} h(x) & \text { if } x \in \bigcup_{n=1}^{\infty}\left[x_{4 n+2 i-4}, x_{4 n+2 i-6}\right], \\ (\operatorname{sgn} f(x))^{i+1} / 2 & \text { if } x=a .\end{cases}
$$

Since $h\left(x_{n}\right)=0$ for each $n \in \mathbb{N} \cup\{0\}$, the function $g_{i}$ is well defined and $g_{i}(b)=0$. We can easily see that $g_{i}(a)=(\operatorname{sgn} f(a))^{i+1} / 2, b \in \mathcal{C}\left(g_{i}\right)$ and

$$
g_{i}\left[(a, c) \cap \mathcal{C}\left(g_{i}\right)\right]=[-1,1] \quad \text { for each } c \in(a, b) .
$$

By Lemma 3.1, $g_{i} \uparrow(a, b] \in \mathcal{S}_{s}$. Hence by (16) and Lemma 3.2, $g_{i} \in \mathcal{S}_{s}$.

Clearly sgn $\circ\left(g_{1} g_{2}\right)=\operatorname{sgn} \circ f\lceil\mathrm{cl} I$. This completes the proof.

Lemma 3.8. Let $I \subset E$ be an open interval such that $I \cap[f=0]=\emptyset$. There are strong Swiatkowski functions $g_{1}, g_{2}: \operatorname{cl} I \rightarrow[-1,1]$ with $\mathrm{sgn} \circ$ $\left(g_{1} g_{2}\right)=\operatorname{sgn} \circ f\left\lceil\operatorname{cl} I\right.$ such that $g_{1}\left[I \cap \mathcal{C}\left(g_{1}\right)\right] \supset(0,1]$ and for $i \in\{1,2\}$, we have: $g_{i}(x)=(\operatorname{sgn} f(x))^{i+1} / 2$ for $x \in$ bd $I$ and bd $I \subset \mathcal{C}\left(g_{i}\right)$.

Proof. Use Lemma 3.5 to construct a strong Świątkowski function $\psi: \operatorname{cl} I \rightarrow[0,1]$ such that $\psi[I]=\psi[I \cap \mathcal{C}(\psi)]=(0,1]$, bd $I \subset \mathcal{C}(\psi)$ and $\psi(x)=|\operatorname{sgn} f(x)| / 2$ for $x \in$ bd $I$. For $i \in\{1,2\}$, define $g_{i}=\psi \cdot(\operatorname{sgn} \circ f)^{i+1}$. It is not hard to see that the functions $g_{1}$ and $g_{2}$ possess all the required properties.

Lemma 3.9. Let $I=(a, b) \subset E$ be an interval such that $f=0$ on $\left(Y_{f} \cap\right.$ $I) \cup\{b\}$. Assume that $I \cap \operatorname{int}[f=0]=\emptyset$ and $a \notin \operatorname{cl}(I \cap[f=0])$. Then there are strong Swiatkowski functions $g_{1}, g_{2}: \operatorname{cl} I \rightarrow[-1,1]$ with $\operatorname{sgn} \circ\left(g_{1} g_{2}\right)=$ sgn $\circ f\left\lceil\operatorname{cl} I\right.$ such that $g_{1}\left[I \cap \mathcal{C}\left(g_{1}\right)\right] \supset(0,1]$ and for $i \in\{1,2\}$, we have: $g_{i}(a)=(\operatorname{sgn} f(a))^{i+1} / 2, g_{i}(b)=0$, and bd $I \subset \mathcal{C}\left(g_{i}\right)$.

Proof. Put

$$
z=\sup \{x \in[a, b]:(a, x) \cap[f=0]=\emptyset\} .
$$

Use Lemma 3.8 to construct strong Świątkowski functions $\psi_{1}, \psi_{2}:[a, z] \rightarrow$ $[0,1]$ with sgn $\circ\left(\psi_{1} \psi_{2}\right)=\operatorname{sgn} \circ f\left\lceil[a, z]\right.$ such that $\psi_{1}\left[(a, z) \cap \mathcal{C}\left(\psi_{1}\right)\right] \supset(0,1]$, and for $i \in\{1,2\}$, we have $\psi_{i}(a)=(\operatorname{sgn} f(a))^{i+1} / 2, \psi_{i}(z)=(\operatorname{sgn} f(z))^{i+1} / 2$ and

$$
a, z \in \mathcal{C}\left(\psi_{i}\right)
$$


If $z \neq b$ and $z \notin \operatorname{cl}((z, b) \cap[f=0])$, then $f(z)=0$. Construct a continuous function $\varphi_{1}:[z, b] \rightarrow[0,1]$ such that $\varphi_{1}[(z, b)]=(0,1]$ and $\varphi_{1}(z)=\varphi_{1}(b)=0$, and use Lemma 3.6 to construct a strong Świątkowski function $\varphi_{2}:[z, b] \rightarrow[-1,1]$ with $\operatorname{sgn} \circ \varphi_{2}=\operatorname{sgn} \circ f\lceil[z, b]$ such that $z, b \in \mathcal{C}\left(\varphi_{2}\right)$.

If $z \in \operatorname{cl}((z, b) \cap[f=0])$, then use Lemma 3.7 to construct strong Świątkowski functions $\bar{g}_{1}, \bar{g}_{2}:[z, b] \rightarrow[-1,1]$ with $\operatorname{sgn} \circ\left(\bar{g}_{1} \bar{g}_{2}\right)=\operatorname{sgn} \circ$ $f \uparrow(z, b]$ such that for $i \in\{1,2\}$, we have $\bar{g}_{i}(b)=0, b \in \mathcal{C}\left(\bar{g}_{i}\right)$, and

$$
\forall_{c \in(z, b)} \bar{g}_{i}\left[(z, c) \cap \mathcal{C}\left(\bar{g}_{i}\right)\right]=[-1,1] .
$$

Fix an $i \in\{1,2\}$. Define the function $g_{i}: \operatorname{cl} I \rightarrow[-1,1]$ by

$$
g_{i}(x)= \begin{cases}\psi_{i}(x) & \text { if } x \in[a, z], \\ \varphi_{i}(x) & \text { if } x \in(z, b] \text { and } z \notin \operatorname{cl}((z, b) \cap[f=0]), \\ \bar{g}_{i}(x) & \text { if } x \in(z, b] \text { and } z \in \operatorname{cl}((z, b) \cap[f=0]) .\end{cases}
$$

We will show that $g_{i} \in \dot{\mathcal{S}}_{s}$. Clearly $g_{i} \uparrow[a, z]=\psi_{i} \in \dot{\mathcal{S}}_{s}$.

If $z \neq b$ and $z \notin \operatorname{cl}((z, b) \cap[f=0])$, then $f(z)=0$ and $g_{i} \uparrow(z, b]=\varphi_{i} \in \mathcal{S}_{s}$. So, $g_{i}(z)=\psi_{i}(z)=\varphi_{i}(z)=0$ and by $(17), z$ is a left-hand side point of continuity of $g_{i}$. Moreover, since $z \in \mathcal{C}\left(\varphi_{i}\right), z$ is a right-hand side point of continuity of $g_{i}$. Hence by Lemma $3.1, g_{i} \in \dot{\mathcal{S}}_{s}$.

If $z \in \operatorname{cl}((z, b) \cap[f=0])$, then $g_{i} \uparrow(z, b]=\bar{g}_{i}$. In this case by (18) and Lemma 3.2 , we obtain $g_{i} \in \dot{\mathcal{S}}_{s}$.

The other requirements of the lemma are evident.

Lemma 3.10. Let $I=(a, b) \subset E$ be an open interval such that $f=0$ on $Y_{f} \cap I$. Assume that $I \subset[f=0]$ or $I \cap \operatorname{int}[f=0]=\emptyset$. There are strong Światkowski functions $g_{1}, g_{2}: \operatorname{cl} I \rightarrow[-1,1]$ with $\operatorname{sgn} \circ\left(g_{1} g_{2}\right)=\operatorname{sgn} \circ f\lceil\operatorname{cl} I$ such that

$$
g_{1}\left[I \cap \mathcal{C}\left(g_{1}\right)\right] \supset(0,1]
$$

and for $i \in\{1,2\}$, we have:

i) $g_{i}(a)=(\operatorname{sgn} f(a))^{i+1} / 2, \quad g_{i}(b)=(\operatorname{sgn} f(b))^{i+1} / 2$,

ii) if $a \in \operatorname{cl}(I \cap[f=0])$, then $g_{i}\left[(a, c) \cap \mathcal{C}\left(g_{i}\right)\right]=[-1,1]$ for each $c \in(a, b)$,

iii) if $a \notin \operatorname{cl}(I \cap[f=0])$, then $a \in \mathcal{C}\left(g_{i}\right)$,

iv) if $b \in \operatorname{cl}(I \cap[f=0])$, then $g_{i}\left[(c, b) \cap \mathcal{C}\left(g_{i}\right)\right]=[-1,1]$ for each $c \in(a, b)$,

v) if $b \notin \operatorname{cl}(I \cap[f=0])$, then $b \in \mathcal{C}\left(g_{i}\right)$,

vi) if $I \cap[f=0] \neq \emptyset$, then $I \cap\left[g_{i}=0\right] \cap \mathcal{C}\left(g_{i}\right) \neq \emptyset$.

Proof. We consider two cases.

Case 1. $I \cap[f=0]=\emptyset$.

This case follows by Lemma 3.8. 
Case $2 . \quad I \cap[f=0] \neq \emptyset$.

Fix a $z \in I \cap[f=0]$. If $a \in \operatorname{cl}(I \cap[f=0])$, then use Lemma 3.7 to construct strong Świątkowski functions $g_{1}, g_{2}:[a, z] \rightarrow[-1,1]$ with sgn $\circ\left(g_{1} g_{2}\right)=\operatorname{sgn} \circ f\left\lceil[a, z]\right.$ such that for $i \in\{1,2\}$, we have $g_{i}(a)=$ $(\operatorname{sgn} f(a))^{i+1} / 2, g_{i}(z)=0$, and

$$
\begin{gathered}
z \in \mathcal{C}\left(g_{i}\right), \\
\forall_{c \in(a, z)} g_{i}\left[(a, c) \cap \mathcal{C}\left(g_{i}\right)\right]=[-1,1] .
\end{gathered}
$$

If $a \notin \operatorname{cl}(I \cap[f=0])$, then use Lemma 3.9 to construct strong Świątkowski functions $g_{1}, g_{2}:[a, z] \rightarrow[-1,1]$ with sgn $\circ\left(g_{1} g_{2}\right)=\operatorname{sgn} \circ f\lceil[a, z]$ such that $g_{1}\left[(a, z) \cap \mathcal{C}\left(g_{1}\right)\right] \supset(0,1]$ and for $i \in\{1,2\}$, we have $g_{i}(a)=(\operatorname{sgn} f(a))^{i+1} / 2$, $g_{i}(z)=0$, and

$$
a, z \in \mathcal{C}\left(g_{i}\right) .
$$

Proceed similarly, using Lemma 3.7 (if $b \in \operatorname{cl}(I \cap[f=0])$ ) or Lemma 3.9 (if $b \notin \operatorname{cl}(I \cap[f=0]))$, to extend the functions $g_{1}$ and $g_{2}$ to whole the interval $\mathrm{cl} I$. Then clearly sgn $\circ\left(g_{1} g_{2}\right)=\operatorname{sgn} \circ f\lceil\operatorname{cl} I$ and the conditions (19) and i) hold.

Fix an $i \in\{1,2\}$. Then $g_{i}(z)=0$. By (22) or (20), $z$ is a left-hand side point of continuity of $g_{i}$. Analogously, $z$ is a right-hand side point of continuity of $g_{i}$. So, the condition vi) is fulfilled and by Lemma $3.1, g_{i} \in \mathcal{S}_{s}$.

If $a \in \operatorname{cl}(I \cap[f=0])$, then by $(21), g_{i}\left[(a, c) \cap \mathcal{C}\left(g_{i}\right)\right]=[-1,1]$ for each $c \in(a, b)$, and the condition ii) holds. If $a \notin \operatorname{cl}(I \cap[f=0])$, then by (22), $a \in \mathcal{C}\left(g_{i}\right)$, and the condition iii) is fulfilled. Similarly we can prove the conditions iv) and v).

Lemma 3.11. Let $I \subset E$ be an open interval. Assume that there is a $G_{\delta^{-}}$set $A \subset[f=0]$ such that

$$
\forall_{a, b \in I} f(a) f(b)<0 \Rightarrow A \cap \mathrm{I}(a, b) \neq \emptyset
$$

and $\operatorname{card}(I \cap A \backslash \operatorname{int}[f=0]) \leq \omega$. Then there are strong Światkowski functions $g_{1}, g_{2}: \operatorname{cl} I \rightarrow[-1,1]$ with $\operatorname{sgn} \circ\left(g_{1} g_{2}\right)=\operatorname{sgn} \circ f\left\lceil\operatorname{cl} I\right.$ such that $g_{1}[I \cap$ $\left.\mathcal{C}\left(g_{1}\right)\right] \supset(0,1]$ and $\left|g_{i}(x)\right| \leq 2^{-1}$ for $i \in\{1,2\}$ and $x \in \operatorname{bd} I$.

Proof. Define

$$
X=\left\{x \in Y_{f} \cap \operatorname{cl} I: f(x) \neq 0\right\} \cup(\operatorname{bdint}[f=0] \cap \operatorname{cl} I),
$$

and let $C=\operatorname{cl} X$. We can easily see that $C$ is nowhere dense. Write the set $C$ as the disjoint union $C=C_{1} \cup C_{2}$, where $C_{1}$ is countable and $C_{2}$ is perfect. Let $\mathcal{P}$ be the family of all components of $I \backslash C_{2}$. Fix a $P \in \mathcal{P}$.

Let $\mathcal{J}_{P}$ be the family of all components of $P \backslash C_{1}$. Since $C_{1}$ is nowhere dense, cl $P=\operatorname{cl} \bigcup \mathcal{J}_{P}$. For each $J \in \mathcal{J}_{P}$ construct functions $g_{1, J}, g_{2, J}: \operatorname{cl} J \rightarrow[-1,1]$, which fulfill the requirements of Lemma 3.10. 
Fix an $i \in\{1,2\}$. Define the function $g_{i, P}: \operatorname{cl} P \rightarrow[-1,1]$ by

$$
g_{i, P}(x)= \begin{cases}g_{i, J}(x) & \text { if } x \in J, J \in \mathcal{J}_{P}, \\ (\operatorname{sgn} f(x))^{i+1} / 2 & \text { otherwise. }\end{cases}
$$

Observe that $g_{i, P}\left\lceil\operatorname{cl} J=g_{i, J} \in \dot{\mathcal{S}}_{s}\right.$ for each $J \in \mathcal{J}_{P}$ and

$$
\begin{aligned}
& \text { if } x \in \operatorname{bd} J \cap \operatorname{bd} J^{\prime} \text { for some } J, J^{\prime} \in \mathcal{J}_{P}, J \neq J^{\prime}, \\
& \text { then } x \in \operatorname{cl}(J \cap[f=0]) \text { or } x \in \operatorname{cl}\left(J^{\prime} \cap[f=0]\right)
\end{aligned}
$$

(since the above assumption yields $x \in X \subset \operatorname{cl}[f=0]$ ).

Now we will show that $g_{i, P} \in \mathcal{S}_{s}$. Let $c, d \in \operatorname{cl} P, c<d$, and $y \in$ $\mathrm{I}\left(g_{i, P}(c), g_{i, P}(d)\right)$. If $c, d \in \mathrm{cl} J$ for some $J \in \mathcal{J}_{P}$, then, since $g_{i, J} \in \mathcal{S}_{s}$, there is an $x_{0} \in(c, d) \cap \mathcal{C}\left(g_{i, P}\right)$ such that $g_{i, P}\left(x_{0}\right)=y$.

In the opposite case there is an $x_{1} \in C_{1} \cap(c, d)$ which is isolated in $C_{1}$; i.e., $x_{1} \in \mathrm{bd} J \cap \mathrm{bd} J^{\prime} \cap(c, d)$ for some $J, J^{\prime} \in \mathcal{J}_{P}, J \neq J^{\prime}$. By (23) and conditions ii) or iv) of Lemma 3.10, we obtain $g_{i, P}\left[(c, d) \cap \mathcal{C}\left(g_{i, P}\right)\right]=[-1,1]$. Hence there is an $x_{0} \in(c, d) \cap \mathcal{C}\left(g_{i, P}\right)$ such that $g_{i, P}\left(x_{0}\right)=y$.

Observe that by condition (19) of Lemma 3.10,

$$
g_{1, P}\left[P \cap \mathcal{C}\left(g_{1, P}\right)\right] \supset(0,1] .
$$

\section{Moreover}

$$
\text { if } P \cap[f=0] \neq \emptyset \text {, then } P \cap\left[g_{i, P}=0\right] \cap \mathcal{C}\left(g_{i, P}\right) \neq \emptyset \text { for } i \in\{1,2\} \text {. }
$$

Indeed, choose an $x_{0} \in P \cap[f=0]$. If $x_{0} \in J$ for some $J \in \mathcal{J}_{P}$, then by condition vi) of Lemma 3.10, we obtain (25). So, assume $x_{0} \in C_{1}$. Then $P \cap C_{1} \neq \emptyset$ and there is an $x_{1} \in P \cap C_{1}$ which is isolated in $C_{1}$. By (23), there is a $J \in \mathcal{J}_{P}$ such that $x_{1} \in \operatorname{cl}(J \cap[f=0])$. Hence by conditions ii) or iv) of Lemma 3.10, we obtain (25).

If $C_{2}=\emptyset$, then $\mathcal{P}=\{I\}$ and $g_{i}=g_{i, I} \in \mathcal{S}_{s}$ for $i \in\{1,2\}$. One can easily show that the other requirements of the lemma are also fulfilled.

So, assume that $C_{2} \neq \emptyset$. Let $\mathcal{P}_{1}, \ldots, \mathcal{P}_{4}$ be pairwise disjoint families of components of $I \backslash C_{2}$ such that $I \backslash C_{2}=\bigcup_{j=1}^{4} \bigcup \mathcal{P}_{j}$ and $C_{2} \subset \operatorname{cl} \bigcup \mathcal{P}_{j}$ for $j \in\{1, \ldots, 4\}$. For $i \in\{1,2\}$ define the function $g_{i}: \operatorname{cl} I \rightarrow[-1,1]$ by

$$
g_{i}(x)= \begin{cases}(-1)^{j} g_{i, P}(x) & \text { if } x \in \operatorname{cl} P \text { and } P \in \mathcal{P}_{j}, j \in\{1,2\}, \\ (-1)^{j} g_{3-i, P}(x) & \text { if } x \in \operatorname{cl} P \text { and } P \in \mathcal{P}_{j}, j \in\{3,4\} \\ (\operatorname{sgn} f(x))^{i+1} / 2 & \text { otherwise }\end{cases}
$$

Then clearly sgn $\circ\left(g_{1} g_{2}\right)=\operatorname{sgn} \circ f\left\lceil\operatorname{cl} I\right.$ and by $(24), g_{1}\left[I \cap \mathcal{C}\left(g_{1}\right)\right] \supset(0,1]$.

Fix an $i \in\{1,2\}$. We can easily see that $\left|g_{i}(x)\right| \leq 2^{-1}$ for $x \in$ bd $I$. So, to complete the proof we should show that $g_{i} \in \mathcal{S}_{s}$.

Let $c, d \in \operatorname{cl} I, c<d$, and $y \in \mathrm{I}\left(g_{i}(c), g_{i}(d)\right)$. We consider two cases.

Case 1. $(c, d) \cap C_{2}=\emptyset$. 
Then there is a $P \in \mathcal{P}$ such that $[c, d] \subset \operatorname{cl} P$. Since $g_{i, P} \in \mathcal{S}_{s}$, there is an $x_{0} \in(c, d) \cap \mathcal{C}\left(g_{i}\right)$ with $g_{i}\left(x_{0}\right)=y$.

Case 2. $(c, d) \cap C_{2} \neq \emptyset$.

Then for each $j \in\{1, \ldots, 4\}$, since $C_{2} \subset \operatorname{cl} \bigcup \mathcal{P}_{j}$, there is an interval $P_{j} \in \mathcal{P}_{j}$ with $P_{j} \subset(c, d)$. Hence by (24), we obtain the following inclusion

$$
g_{i}\left[(c, d) \cap \mathcal{C}\left(g_{i}\right)\right] \supset g_{i}\left[\bigcup_{j=1}^{4} P_{j} \cap \mathcal{C}\left(g_{i}\right)\right] \supset[-1,1] \backslash\{0\} .
$$

Since $A \cap C_{2} \subset \operatorname{cl} I \cap A \backslash \operatorname{int}[f=0]$ is a countable $G_{\delta^{-}}$-set, it is nowhere dense in $C_{2}$. So, there is an interval $\left(c^{\prime}, d^{\prime}\right) \subset(c, d)$ with $\left(c^{\prime}, d^{\prime}\right) \cap C_{2} \neq \emptyset$ such that $\left(c^{\prime}, d^{\prime}\right) \cap A \cap C_{2}=\emptyset$. Hence the function $f$ changes its sign in $\left(c^{\prime}, d^{\prime}\right)$ or $\left(c^{\prime}, d^{\prime}\right) \cap \operatorname{int}[f=0] \neq \emptyset$. In both cases there is a $P \in \mathcal{P}$ such that $P \subset$ $\left(c^{\prime}, d^{\prime}\right) \subset(c, d)$ and $P \cap[f=0] \neq \emptyset$. Then by $(25), g_{i}\left[(c, d) \cap \mathcal{C}\left(g_{i}\right)\right]=[-1,1]$, whence $g_{i}\left(x_{0}\right)=y$ for some $x_{0} \in(c, d) \cap \mathcal{C}\left(g_{i}\right)$.

\section{Main results}

Proposition 4.1. Assume that there is a $G_{\delta}$-set $A \subset[f=0]$ such that

$$
\forall_{a, b \in E} f(a) f(b)<0 \Rightarrow A \cap \mathrm{I}(a, b) \neq \emptyset .
$$

Then there are functions $g_{1}, \ldots, g_{4} \in \mathcal{S}_{s}$ such that $f=g_{1} \ldots g_{4}$.

Proof. Define

$$
C=E \backslash \bigcup\{(a, b): \operatorname{card}((a, b) \cap A \backslash \operatorname{int}[f=0]) \leq \omega\} .
$$

First we will show that

$$
\begin{aligned}
& \text { there are strong Świątkowski functions } g_{1}, g_{2} \\
& \text { with sgn } \circ\left(g_{1} g_{2}\right)=\operatorname{sgn} \circ f \text {. }
\end{aligned}
$$

If $C=\emptyset$, then (26) follows by Lemma 3.11 .

So, assume that $C \neq \emptyset$. Then $C$ is perfect and nowhere dense. Let $\mathcal{I}_{1}, \ldots, \mathcal{I}_{4}$ be pairwise disjoint families of components of $E \backslash C$ such that $E \backslash C=\bigcup_{j=1}^{4} \bigcup \mathcal{I}_{j}$ and $C \subset \operatorname{cl} \bigcup \mathcal{I}_{j}$ for $j \in\{1, \ldots, 4\}$. Put $\mathcal{I}=\mathcal{I}_{1} \cup \cdots \cup \mathcal{I}_{4}$ and

$$
A_{1}=A \cap C \backslash \bigcup_{I \in \mathcal{I}} \mathrm{bd} I .
$$

Since $A$ is a $G_{\delta^{-}}$set, $A_{1}$ is a $G_{\delta^{-}}$set, too. Then $C \backslash A_{1}$ is an $F_{\sigma^{-}}$-set, whence there is a sequence $\left(F_{n}\right)$ consisting of closed sets such that

$$
C \backslash A_{1}=\bigcup_{n \in \mathbb{N}} F_{n} .
$$


Define $F_{0}^{\prime}=\emptyset$. For each $n \in \mathbb{N}$, use four times Lemma 3.4 to construct a sequence of sets $\left(F_{n}^{\prime}\right)$ and a sequence of families of intervals $\left(\mathcal{J}_{n}^{\prime}\right)$ such that

$$
\begin{gathered}
\mathcal{J}_{n}^{\prime}=\bigcup_{j=1}^{4} \mathcal{J}_{j, n}^{\prime}, \\
F_{n}^{\prime}=F_{n} \cup \bigcup_{k<n}\left(F_{k}^{\prime} \cup \bigcup_{I \in \mathcal{J}_{k}^{\prime}} \mathrm{bd} I\right)
\end{gathered}
$$

and for $j \in\{1, \ldots, 4\}$,

$$
\mathcal{J}_{j, n}^{\prime} \subset \mathcal{I}_{j}
$$

for each $I \in \mathcal{I}_{j}$, if $F_{n}^{\prime} \cap$ bd $I \neq \emptyset$, then $I \in \mathcal{J}_{j, n}^{\prime}$,

for each $c \in F_{n}^{\prime}$, if $c$ is a right-hand (left-hand) limit point of $C$, then $c$ is a right-hand (left-hand) limit point of the union $\bigcup \mathcal{J}_{j, n}^{\prime}$,

$$
\operatorname{cl} \bigcup \mathcal{J}_{j, n}^{\prime} \subset F_{n}^{\prime} \cup \bigcup_{J \in \mathcal{J}_{j, n}^{\prime}} \operatorname{cl} J .
$$

(Observe that by (34), for each $k<n$, the set $F_{k}^{\prime} \cup \bigcup_{I \in \mathcal{J}_{k}^{\prime}}$ bd $I$ is closed. So by (30), the set $F_{n}^{\prime}$ is also closed and $F_{n}^{\prime} \subset C \backslash A_{1}$.)

Fix an $I \in \mathcal{I}$. Construct strong Świątkowski functions $g_{1, I}, g_{2, I}: \operatorname{cl} I \rightarrow$ $[-1,1]$ such that $\operatorname{sgn} \circ\left(g_{1, I} g_{2, I}\right)=\operatorname{sgn} \circ f\left\lceil\operatorname{cl} I, g_{1, I}\left[I \cap \mathcal{C}\left(g_{1, I}\right)\right] \supset(0,1]\right.$, and $\left|g_{i, I}(x)\right| \leq 2^{-1}$ for $i \in\{1,2\}$ and $x \in \mathrm{bd} I$. (We use Lemma 3.11.) Put

$$
n_{I}=\min \left\{n \in \mathbb{N}: I \in \mathcal{J}_{n}^{\prime}\right\},
$$

and observe that by (32), $\bigcup_{n \in \mathbb{N}} \cup \mathcal{J}_{n}^{\prime}=E \backslash C$, whence $n_{I}$ is well defined.

Fix an $i \in\{1,2\}$. Define the function $g_{i}: E \rightarrow\left[-2^{-1}, 2^{-1}\right]$ by

$$
g_{i}(x)= \begin{cases}0 & \text { if } x \in A_{1}, \\ (-1)^{j} 2^{-n_{I}} g_{i, I}(x) & \text { if } x \in \operatorname{cl} I \text { and } I \in \mathcal{I}_{j}, j \in\{1,2\}, \\ (-1)^{j} 2^{-n_{I}} g_{3-i, I}(x) & \text { if } x \in \operatorname{cl} I \text { and } I \in \mathcal{I}_{j}, j \in\{3,4\}, \\ 2^{-n}(\operatorname{sgn} f(x))^{i+1} & \text { if } x \in F_{n}^{\prime} \backslash\left(\bigcup_{I \in \mathcal{I}} \mathrm{bd} I \cup F_{n-1}^{\prime}\right), n \in \mathbb{N} .\end{cases}
$$

First we will show that $A_{1} \subset \mathcal{C}\left(g_{i}\right)$.

Take an $x_{0} \in A_{1}$ and let $\varepsilon>0$. Choose $n_{0} \in \mathbb{N}$ such that $2^{-n_{0}}<\varepsilon$ and put $\delta \stackrel{\text { df }}{=} \operatorname{dist}\left(\operatorname{cl} \bigcup \mathcal{J}_{n_{0}}^{\prime}, x_{0}\right)$. Since by (34), (30), (27), and (28),

$$
\begin{aligned}
A_{1} \cap \operatorname{cl} \bigcup \mathcal{J}_{j, n_{0}}^{\prime} & \subset\left(A_{1} \cap F_{n_{0}}^{\prime}\right) \cup\left(A_{1} \cap \bigcup_{J \in \mathcal{J}_{j, n_{0}}^{\prime}} \operatorname{cl} J\right) \\
& \subset\left(A_{1} \cap \bigcup_{n \leq n_{0}} F_{n}\right) \cup\left(\left(C \backslash \bigcup_{I \in \mathcal{I}} \mathrm{bd} I\right) \cap \bigcup_{J \in \mathcal{I}} \operatorname{cl} J\right)=\emptyset,
\end{aligned}
$$


we have $x \notin \operatorname{cl} \bigcup \mathcal{J}_{j, n_{0}}^{\prime}$ and $\delta>0$.

Observe that by (33), $F_{n_{0}}^{\prime} \subset \operatorname{cl} \bigcup \mathcal{J}_{n_{0}}^{\prime}$. If $\left|x-x_{0}\right|<\delta$, then $x \notin \operatorname{cl} \bigcup \mathcal{J}_{n_{0}}^{\prime}$, whence

$$
\left|g_{i}(x)-g_{i}\left(x_{0}\right)\right|=\left|g_{i}(x)\right| \leq 2^{-n_{0}}<\varepsilon .
$$

So, $x_{0} \in \mathcal{C}\left(g_{i}\right)$.

Now we will prove that

$$
\begin{aligned}
\forall_{n \in \mathbb{N}} \forall_{\delta>0} & \left(x \in F_{n}^{\prime} \backslash\{\sup I: I \in \mathcal{I}\}\right. \\
& \left.\Rightarrow g_{i}\left[(x-\delta, x) \cap \mathcal{C}\left(g_{i}\right)\right] \supset\left[-2^{-n}, 2^{-n}\right]\right) .
\end{aligned}
$$

Let $n \in \mathbb{N}, \delta>0$ and $x \in F_{n}^{\prime} \backslash\{\sup I: I \in \mathcal{I}\}$. Then for $j \in\{1, \ldots, 4\}$, by (33), there is an $I_{j} \in \mathcal{J}_{j, n}^{\prime}$ with $I_{j} \subset(x-\delta, x)$. Notice that $\max \left\{n_{I_{j}}\right.$ : $j \in\{1, \ldots, 4\}\} \leq n$. So,

$$
g_{i}\left[(x-\delta, x) \cap \mathcal{C}\left(g_{i}\right)\right] \supset \bigcup_{j=1}^{4} g_{i}\left[I_{j} \cap \mathcal{C}\left(g_{i}\right)\right] \supset\left[-2^{-n}, 2^{-n}\right] \backslash\{0\} .
$$

Since $x \notin\{\sup I: I \in \mathcal{I}\}$, we have $\operatorname{card}\left((x-\delta, x) \cap A_{1}\right)>\omega$. Hence

$$
\emptyset \neq(x-\delta, x) \cap A_{1} \subset(x-\delta, x) \cap \mathcal{C}\left(g_{i}\right) \cap\left[g_{i}=0\right]
$$

and finally

$$
g_{i}\left[(x-\delta, x) \cap \mathcal{C}\left(g_{i}\right)\right] \supset\left[-2^{-n}, 2^{-n}\right] .
$$

Similarly we can prove that

$$
\forall_{n \in \mathbb{N}} \forall_{\delta>0}\left(x \in F_{n}^{\prime} \backslash\{\inf I: I \in \mathcal{I}\} \Rightarrow g_{i}\left[(x, x+\delta) \cap \mathcal{C}\left(g_{i}\right)\right] \supset\left[-2^{-n}, 2^{-n}\right]\right) .
$$

Now we will show that $g_{i} \in \dot{\mathcal{S}}_{s}$. Let $c, d \in E, c<d$, and $y \in \mathrm{I}\left(g_{i}(c), g_{i}(d)\right)$. Assume that $g_{i}(c)<g_{i}(d)$. (The other case is similar.) If $c, d \in \operatorname{cl} I$ for some $I \in \mathcal{I}$, then since $g_{1, I}, g_{2, I} \in \mathcal{S}_{s}$, there is an $x_{0} \in(c, d) \cap \mathcal{C}\left(g_{i}\right)$ with $g_{i}\left(x_{0}\right)=y$. So, assume that the opposite case holds.

Assume that $y \geq 0$. (The case $y<0$ is analogous.) Then $g_{i}(d)>0$, whence $d \notin A_{1}$. We consider two cases.

Case 1. $d \notin \bigcup_{n \in \mathbb{N}} F_{n}^{\prime}$ or $d \in\{\sup I: I \in \mathcal{I}\}$.

Then there is an $I \in \mathcal{I}$ such that $d \in \operatorname{cl} I$ and $c \notin \operatorname{cl} I$. If $y \in$ $\mathrm{I}\left(g_{i}(\inf I), g_{i}(d)\right)$, then, since $g_{1, I}, g_{2, I} \in \mathcal{S}_{s}$, there is an $x_{0} \in(\inf I, d) \cap$ $\mathcal{C}\left(g_{i}\right) \subset(c, d) \cap \mathcal{C}\left(g_{i}\right)$ with $g_{i}\left(x_{0}\right)=y$.

Now let $y \in\left[0, g_{i}(\inf I)\right]$. By $(30)$, since $I \in \mathcal{J}_{n_{I}}^{\prime}$, we have inf $I \in F_{n_{I}+1}^{\prime}$. By (35),

$$
y \in\left[0, g_{i}(\inf I)\right] \subset\left[-2^{-n_{I}-1}, 2^{-n_{I}-1}\right] \subset g_{i}\left[(c, \inf I) \cap \mathcal{C}\left(g_{i}\right)\right] .
$$

So, there is an $x_{0} \in(c, \inf I) \cap \mathcal{C}\left(g_{i}\right) \subset(c, d) \cap \mathcal{C}\left(g_{i}\right)$ with $g_{i}\left(x_{0}\right)=y$.

Case 2. $d \in \bigcup_{n \in \mathbb{N}} F_{n}^{\prime} \backslash\{\sup I: I \in \mathcal{I}\}$. 
Then $d \in F_{n}^{\prime} \backslash F_{n-1}^{\prime}$ for some $n \in \mathbb{N}$. By (35),

$$
y \in\left[0, g_{i}(d)\right) \subset\left[-2^{-n}, 2^{-n}\right] \subset g_{i}\left[(c, d) \cap \mathcal{C}\left(g_{i}\right)\right] .
$$

Consequently, there is an $x_{0} \in(c, d) \cap \mathcal{C}\left(g_{i}\right)$ with $g_{i}\left(x_{0}\right)=y$. It follows that $g_{i} \in \mathcal{S}_{s}$.

It is not hard to see that sgn $\circ\left(g_{1} g_{2}\right)=\operatorname{sgn} \circ f$. This completes the proof of (26).

Now define the function $\tilde{f}: E \rightarrow \mathbb{R}$ by

$$
\tilde{f}(x)=\left\{\begin{array}{cl}
\frac{f}{g_{1} g_{2}}(x) & \text { if } f(x) \neq 0, \\
1 & \text { otherwise. }
\end{array}\right.
$$

Notice that $\tilde{f}$ is cliquish. Indeed, it is obvious that

$$
\mathcal{C}(\tilde{f}) \supset \mathcal{C}(f) \cap \mathcal{C}\left(g_{1}\right) \cap \mathcal{C}\left(g_{2}\right) \cap U
$$

where

$$
U \stackrel{\mathrm{df}}{=} \operatorname{int}[f=0] \cup \operatorname{int}[f \neq 0]
$$

By assumption, the set $[f=0]$ is simply open. So, $\mathbb{R} \backslash U=\operatorname{bd}[f=0]$ is nowhere dense and $U$ is residual. Since the sets $\mathcal{C}(f), \mathcal{C}\left(g_{1}\right)$, and $\mathcal{C}\left(g_{2}\right)$ are also residual, so is the $\operatorname{set} \mathcal{C}(\tilde{f})$.

Clearly $\tilde{f}>0$ on $E$. So, the function $\ln \circ \tilde{f}: E \rightarrow \mathbb{R}$ is cliquish. By [7, Corollary II.3.4], there are functions $h_{1}, h_{2} \in \mathcal{S}_{s}$ such that $\ln \circ \tilde{f}=h_{1}+h_{2}$.

Define $g_{3}=\exp \circ h_{1}$ and $g_{4}=\exp \circ h_{2}$. By Lemma 3.3, $g_{3}, g_{4} \in \dot{\mathcal{S}}_{s}$. Clearly

$$
f=g_{1} g_{2} \cdot \tilde{f}=g_{1} g_{2}\left(\exp \circ h_{1}\right)\left(\exp \circ h_{2}\right)=g_{1} \ldots g_{4},
$$

which completes the proof.

Remark 4.1. In Theorem 4.2, we do not assume that conditions (7)-(9) are fulfilled.

Theorem 4.2. Let $f: \mathbb{R} \rightarrow \mathbb{R}$. The following conditions are equivalent:

i) there are functions $g_{1}, \ldots, g_{4} \in \mathcal{S}_{s}$ such that $f=g_{1} \ldots g_{4}$,

ii) there is a $k \in \mathbb{N}$ and functions $g_{1}, \ldots, g_{k} \in \mathcal{S}_{s}$ such that $f=g_{1} \ldots g_{k}$,

iii) the function $f$ is cliquish, the set $[f=0]$ is simply open, and there is $a G_{\delta}$-set $A \subset[f=0]$ such that for all $a, b \in \mathbb{R}$, if $f(a) f(b)<0$, then $A \cap \mathrm{I}(a, b) \neq \emptyset$.

Proof. The implication i) $\Rightarrow$ ii) is evident.

ii) $\Rightarrow$ iii). Since $f \in \mathcal{S}_{s}$, it is cliquish as well. By [3], the set $[f=0]$ is simply open. (Cf. also [8, Theorem] or [2].) 
For $i \in\{1, \ldots, k\}$, define

$$
\tilde{g}_{i}=\min \left\{\max \left\{g_{i},-1\right\}, 1\right\} .
$$

Then the function $\tilde{g}_{i}$ is bounded. One can readily verify that $\tilde{g}_{i} \in \mathcal{S}_{s}$.

Put $\tilde{f}=\tilde{g}_{1} \ldots \tilde{g}_{k}$. Observe that $\operatorname{sgn} \circ \tilde{f}=\operatorname{sgn} \circ f$. Define a $G_{\delta}$-set

$$
A \stackrel{\text { df }}{=} \bigcap_{n=1}^{\infty} \operatorname{int} \tilde{f}^{-1}\left(\left(-n^{-1}, n^{-1}\right)\right)=[\tilde{f}=0] \cap \mathcal{C}(\tilde{f}) \subset[f=0] .
$$

Let $I \subset \mathbb{R}$ be an interval in which $f$ changes its sign. Then at least one of the functions $\tilde{g}_{1}, \ldots, \tilde{g}_{k}$, say $\tilde{g}_{1}$, changes its sign in $I$, too. Since $\tilde{g}_{1} \in \mathcal{S}_{s}$, there is an $x_{0} \in I \cap \mathcal{C}\left(\tilde{g}_{1}\right)$ such that $\tilde{g}_{1}\left(x_{0}\right)=0$. The functions $\tilde{g}_{1}, \ldots, \tilde{g}_{k}$ are bounded, so $x_{0} \in \mathcal{C}(\tilde{f})$, and finally $x_{0} \in I \cap[\tilde{f}=0] \cap \mathcal{C}(\tilde{f})=I \cap A$.

iii) $\Rightarrow$ i). Put $E=[-\pi / 2, \pi / 2]$. Define the function $\tilde{f}: E \rightarrow \mathbb{R}$ by

$$
\tilde{f}(x)=\left\{\begin{array}{cl}
(f \circ \tan )(x) & \text { if } x \in(-\pi / 2, \pi / 2), \\
0 & \text { if } x \in\{-\pi / 2, \pi / 2\} .
\end{array}\right.
$$

Then clearly $\tilde{A} \stackrel{\mathrm{df}}{=} \arctan [A] \subset[\tilde{f}=0]$ is a $G_{\delta^{-}}$set, and for each interval $I \subset E$, if the function $\tilde{f}$ changes its sign in $I$, then $I \cap \tilde{A} \neq \emptyset$. So, by Proposition 4.1 , there are functions $\tilde{g}_{1}, \ldots, \tilde{g}_{4} \in \mathcal{S}_{s}$ such that $\tilde{f}=\tilde{g}_{1} \ldots \tilde{g}_{4}$. For $i \in\{1, \ldots, 4\}$ define $g_{i}=\tilde{g}_{i} \circ$ arctan and notice that by Lemma 3.3, $g_{i} \in \mathcal{S}_{s}$. Clearly

$$
f=\tilde{f} \circ \arctan =\left(\tilde{g}_{1} \circ \arctan \right) \ldots\left(\tilde{g}_{4} \circ \arctan \right)=g_{1} \ldots g_{4},
$$

which completes the proof.

\section{References}

[1] Biswas, N., On some mappings in topological spaces, Bull. Calcutta Math. Soc. 61 (1969), 127-135.

[2] Borsík, J., Products of simply continuous and quasicontinuous functions, Math. Slovaca 45(4), (1995), 445-452.

[3] Grande, Z., Sur le fonctions cliquish, Časopis Pěst. Mat. 110 (1985), 225-236.

[4] Kempisty, S., Sur les fonctions quasicontinues, Fund. Math. 19 (1932), 184-197.

[5] Kucner, J., Pawlak, R. J., On local characterization of the strong Światkowski property for a function $f:[a, b] \rightarrow \mathbb{R}$, Real Anal. Exchange 28(2) (2002/03), 563-572.

[6] Maliszewski, A., On the limits of strong Światkowski functions, Zeszyty Nauk. Politech. Łódz. Mat. 27(719) (1995), 87-93.

[7] Maliszewski, A., Darboux Property and Quasi-Continuity. A Uniform Approach, WSP, Słupsk, 1996.

[8] Natkaniec, T., Products of quasi-continuous functions, Math. Slovaca 40(4) (1990), 401-405.

[9] Szczuka, P, Products of strong Światkowski functions, Proceedings of International Conference on Real Functions Theory, Rowy, 2003.

[10] Thielman, H. P., Types of functions, Amer. Math. Monthly 60(3) (1953), 156-161. 
PAUlina SzcZuKa

KAZIMIERZ WIELKI UNIVERSITY

Pl. Weyssenhoffa 11

85-072 Bydgoszcz, Poland

E-MAIL:PAULINA@AB.EDU.PL 